\title{
Modelling of the effects of Lotronex and Zelnorm on intestinal propulsion
}

\author{
R. Miftahof \\ Division of Applied Mathematics, KAIST, Daejeon, Republic of Korea
}

\begin{abstract}
A mathematical model of a segment of the gut to simulate propulsion of a solid pellet (bolus) under physiological conditions and after administration of the drugs - Lotronex (GSK) and Zelnorm (Novartis, AB) - is developed. The gut containing a pellet is represented as a thin deformable soft biological shell with the pellet modeled as a non-deformable solid. Mechanical properties of the gut wall satisfy the general type of nonlinear orthotropy and deformations are finite. Bolus propulsion is numerically simulated by generation and propagation of an electromechanical wave of excitation along the wall. Pharmacological manipulation is applied to model the effects of Lotronex and Zelnorm on the dynamics of bolus progression. The results lead to new quantitative insights into the complex spatio-temporal patterns of gastrointestinal transit. It is demonstrated that the reciprocal relationship in contraction of the longitudinal and circular smooth muscle syncytia is necessary to provide the "mixing" type of movements during the preparatory phase of propulsion. Strong simultaneous contractions of both smooth muscle layers are required to expel the "mixed" pellet from the segment. The model is implemented as an interactive software system, Gut Discovery (C), and accurately predicts the effects of drugs on gut motility.
\end{abstract}

Keywords: pellet propulsion, mathematical model of the gut, effects of drugs.

\section{Introduction}

Despite considerable experimental progress in the past decade in the understanding of the intricate biological mechanisms involved in the regulation of gastrointestinal transit, our conceptual knowledge of the basic processes responsible for intestinal movements is still lacking. This is mainly because of 
the complexity of the analysis that requires considering biological phenomena simultaneously at the macroscopic and microscopic levels. To solve the above problems mathematical and computer models need to be developed that would couple multilevel dynamics simulations. Such models would have far-reaching applications in the study of pathogenesis of gastrointestinal dysfunction, to validate potential drug targets and to assess therapeutic efficacy and safety of drugs.

The aim of this investigation was to develop a mathematical model of a segment of the gut and to simulate numerically peristaltic propulsion of a solid pellet (bolus) under normal physiological conditions and after administration of the two drugs - Lotronex (GSK) and Zelnorm (Novartis, AB). The present work is a continuation of our previous research [1-3] and the reader is advised to consult our earlier publications on this matter.

\section{Mathematical model}

A new class of mathematical models to simulate the gastrointestinal tract has been developed. It comprises: a biomechanical model of a functional unit (FU), [2], and a model of an artificial pellet as a rigid non-deformable body.

The mechanical principles of organization of the FU satisfy the small intestinal segment dynamics:

$$
\begin{gathered}
\gamma_{0}\left(\frac{\partial v_{r}}{\partial t}-\frac{v_{s}^{2}}{r}\right)=\frac{\partial}{\partial \tilde{s}_{1}}\left[\left(\kappa \frac{\partial\left(\lambda_{c}-1\right)}{\partial t}+T^{a}\left(\lambda_{c}\right)+T^{p}\left(\lambda_{c}, \lambda_{l}\right)\right) e_{1 r} \sqrt{g_{22}}\right]+ \\
\frac{\partial}{\partial \tilde{s}_{2}}\left[\left(\kappa \frac{\partial\left(\lambda_{l}-1\right)}{\partial t}+T^{a}\left(\lambda_{l}\right)+T^{p}\left(\lambda_{c}, \lambda_{l}\right)\right) e_{2 r} \sqrt{g_{11}}\right]+p \sqrt{g} n_{r,} \\
\gamma_{0}\left(\frac{\partial v_{s}}{\partial t}-\frac{v_{s} v_{r}}{r}\right)=\frac{\partial}{\partial \tilde{s}_{1}}\left[\left(\kappa \frac{\partial\left(\lambda_{c}-1\right)}{\partial t}+T^{a}\left(\lambda_{c}\right)+T^{p}\left(\lambda_{c}, \lambda_{l}\right)\right) e_{1 s} \sqrt{g_{22}}\right]+ \\
\frac{\partial}{\partial \tilde{s}_{2}}\left[\left(\kappa \frac{\partial\left(\lambda_{l}-1\right)}{\partial t}+T^{a}\left(\lambda_{l}\right)+T^{p}\left(\lambda_{c}, \lambda_{l}\right)\right) e_{2 s} \sqrt{g_{11}}\right]+p \sqrt{g} n_{s,} \\
\gamma_{0} \frac{\partial v_{z}}{\partial t}=\frac{\partial}{\partial \tilde{s}_{1}}\left[\left(\kappa \frac{\partial\left(\lambda_{c}-1\right)}{\partial t}+T^{a}\left(\lambda_{c}\right)+T^{p}\left(\lambda_{c}, \lambda_{l}\right)\right) e_{1 z} \sqrt{g_{22}}\right]+ \\
\frac{\partial}{\partial \tilde{s}_{2}}\left[\left(\kappa \frac{\partial\left(\lambda_{l}-1\right)}{\partial t}+T^{a}\left(\lambda_{l}\right)+T^{p}\left(\lambda_{c}, \lambda_{l}\right)\right) e_{2 z} \sqrt{g_{11}}\right]+p \sqrt{g} n_{z,}
\end{gathered}
$$

Here, the following notations are used: the radial $\left(v_{r}\right)$, circumferential $\left(v_{s}\right)$ and longitudinal components $\left(v_{z}\right)$ of the wall velocity vector; ; $\lambda_{l} \lambda_{c}$ - the rate of elongation (hereafter, the subscripts $(l)$ and $(c)$ are related to the longitudinal and circular muscle layers, respectively); $g_{i j}$ - the components and determinant of the 
fundamental tensor; $\gamma_{0}$ - the linear density of a biomaterial in an undeformed state; $T^{p}, T^{a}$ - the passive and active components, respectively, of the total force $\left(T_{c, l}\right)$ of the wall; $\kappa$-rheological parameter; $p$-intraluminal pressure; $\varphi_{l}, \varphi_{c}-$ the electrical waves of depolarization of the longitudinal and circular muscle layers; $\tilde{s}_{1}, \tilde{s}_{2}$ - the Lagrangian coordinates of the bioshell.

The passive $\left(T_{c, l}^{p}\right)$ components are calculated from:

$$
T_{c, l}^{p}=\frac{\partial \gamma_{0} W}{\partial\left(\lambda_{c, l}-1\right)}
$$

where: $W$ - the strain energy density function of the connective tissue and passive muscular components:

$$
\begin{aligned}
\gamma_{0} W= & \frac{1}{2}\left[c_{1}\left(\lambda_{l}-1\right)^{2}+2 c_{3}\left(\lambda_{l}-1\right)\left(\lambda_{c}-1\right)+c_{2}\left(\lambda_{c}-1\right)^{2}\right. \\
& \left.+c_{14} \exp \left(c_{4}\left(\lambda_{l}-1\right)^{2}+c_{5}\left(\lambda_{c}-1\right)^{2}+2 c_{6}\left(\lambda_{l}-1\right)\left(\lambda_{c}-1\right)\right)\right] .
\end{aligned}
$$

For the active force $\left(T_{c, l}^{a}\right)$ components, we assume:

$$
T_{c, l}^{a}=\left\{\begin{array}{c}
0, \quad\left[\mathrm{Ca}^{2+}\right] \leq 0.1 \mu M \\
\left.c_{7}+c_{8}\left[\mathrm{Ca}^{2+}\right]^{4}+c_{9}\left[\mathrm{Ca}^{2+}\right]^{3}+c_{10}\left[\mathrm{Ca}^{2+}\right\}\right]^{2} \\
+c_{11}\left[\mathrm{Ca}^{2+}\right], \quad 0.1<\left[\mathrm{Ca}^{2+}\right] \leq 1 \mu M \\
\max T^{a}, \quad\left[\mathrm{Ca}^{2+}\right]>1 \mu M
\end{array},\right.
$$

where: $T^{a}$ - active force generated by smooth muscle syncytium; $c_{1-11}-$ mechanical constants.

The mechanical activity of the FU is under the control of a "pacemaker cell" that is represented by interstitial cells of Cajal (ICC-MP). The latter generate high amplitude action potentials at constant frequency and amplitude.

The dynamics of propagation of the electrical wave, $\varphi_{l}$, along the aniso-tropic longitudinal muscle layer is defined as:

$$
C_{m} \frac{\partial \varphi_{l}}{\partial t}=I_{m 1}\left(\tilde{s}_{1}, \tilde{s}_{2}\right)+I_{m 2}\left(\tilde{s}_{1}-\tilde{s}_{1}^{\prime}, \tilde{s}_{2}-\tilde{s}_{2}^{\prime}\right)+I_{\text {ionic }}^{*}
$$

where: $I_{m 1}, I_{m 2}-$ the transmembrane ion currents per unit volume are calculated from: 


$$
\begin{aligned}
& I_{m 1}\left(\tilde{s}_{1}, \tilde{s}_{2}\right)=M_{v s}\left\{-\frac{2\left(\mu_{\tilde{s}_{1}}-\mu_{\tilde{s}_{2}}\right)}{\left(1+\mu_{\tilde{s}_{1}}\right)\left(1+\mu_{\tilde{s}_{1}}\right)} \arctan \left(\frac{d \tilde{s}_{1}}{d \tilde{s}_{2}} \sqrt{\frac{G_{\tilde{s}_{2}}}{G_{\tilde{s}_{1}}}}\right)\right. \\
& \left.+\frac{g_{0, \tilde{s}_{2}}^{*}}{G_{\tilde{s}_{1}}}\right\}\left(\frac{\partial}{\partial \tilde{s}_{1}}\left(\frac{g_{0, \tilde{s}_{1}}^{*}}{\lambda_{c}} \frac{\partial \varphi_{l}}{\partial \tilde{s}_{1}}\right)+\frac{\partial}{\partial \tilde{s}_{2}}\left(\frac{g_{0, \tilde{s}_{2}}^{*}}{\lambda_{l}} \frac{\partial \varphi_{l}}{\partial \tilde{s}_{2}}\right)\right), \\
& I_{m 2}\left(\tilde{s}_{1}, \tilde{s}_{2}\right)=M_{v s} \iint_{S} \frac{\mu_{\tilde{s}_{1}}-\mu_{\tilde{s}_{2}}}{2 \pi\left(1+\mu_{\tilde{s}_{1}}\right)\left(1+\mu_{\tilde{s}_{1}}\right)} \\
& \times \frac{\left(\tilde{s}_{2}-\tilde{s}_{2}^{\prime}\right) / G_{\tilde{s}_{2}}-\left(\tilde{s}_{1}-\tilde{s}_{1}^{\prime}\right) / G_{\tilde{s}_{1}}}{\left[\left(\tilde{s}_{1}-\tilde{s}_{1}^{\prime}\right) / G_{\tilde{s}_{1}}-\left(\tilde{s}_{2}-\tilde{s}_{2}^{\prime}\right) / G_{\tilde{s}_{2}}\right]^{2}} \\
& \times\left(\frac{\partial}{\partial \tilde{s}_{1}}\left(\frac{g_{0, \tilde{s}_{1}}^{*}}{\lambda_{c}} \frac{\partial \varphi_{l}}{\partial \tilde{s}_{1}}\right)+\frac{\partial}{\partial \tilde{s}_{2}}\left(\frac{g_{0, \tilde{s}_{2}}^{*}}{\lambda_{l}} \frac{\partial \varphi_{l}}{\partial \tilde{s}_{2}}\right)\right) d \tilde{s}_{1}^{\prime} d \tilde{s}_{2}^{\prime},
\end{aligned}
$$

Here:

$$
\begin{aligned}
& \mu_{\tilde{s}_{1}}=g_{0, \tilde{s}_{1}}^{*} / g_{i, \tilde{s}_{1}}^{*}, \quad \mu_{\tilde{s}_{2}}=g_{0, \tilde{s}_{2}}^{*} / g_{i, \tilde{s}_{2}}^{*}, \\
& G_{\tilde{s}_{1}}=\frac{g_{0, \tilde{s}_{1}}^{*}+g_{i, \tilde{s}_{1}}^{*}}{\lambda_{c}}, G_{\tilde{s}_{2}}=\frac{g_{0, \tilde{s}_{2}}^{*}+g_{i, \tilde{s}_{2}}^{*}}{\lambda_{l}}, G=\sqrt{G_{\tilde{s}_{1}} G_{\tilde{s}_{2}}},
\end{aligned}
$$

and the following notations are accepted: $C_{m}-$ the capacitance of smooth muscle; $g_{i, \tilde{s}_{1}}^{*}, g_{i, \tilde{s}_{2}}^{*}, g_{0, \tilde{s}_{1}}^{*}, g_{0, \tilde{s}_{2}}^{*}$ - the maximal intracellular (the subscript $(i)$ ) and interstitial space (the subscript $(0)$ ) conductivity of the longitudinal and circular muscle layers in the longitudinal and circumferential directions, respectively; $M_{v s}$ - the membrane volume-to-surface ratio, $I_{i o n i c}^{*}-$ the total ionic current:

$$
\begin{aligned}
& I_{\text {ionic }}^{*}=\bar{g}_{N a} m^{* 3} h^{*}\left(\varphi_{(c, l)}-\bar{\varphi}_{N a}\right)+\bar{g}_{K} n^{* 4}\left(\varphi_{(c, l)}-\bar{\varphi}_{K}\right) \\
& +\bar{g}_{\theta}\left(\varphi_{(c, l)}-\bar{\varphi}_{\theta}\right) .
\end{aligned}
$$

$\bar{g}_{N a}, \bar{g}_{K}, \bar{g}_{\theta}$ are the maximal conductances; $m^{*}, h^{*}$, and $n^{*}$ are the probabilities of opening of the ion gates, and $\bar{\varphi}_{\mathrm{Na}}, \bar{\varphi}_{\mathrm{K}}, \bar{\varphi}_{\theta}$ are the reversal potentials of $\mathrm{Na}^{+}$, $\mathrm{K}^{+}$, and $\mathrm{Cl}^{-}$currents, respectively, in smooth muscles.

The dynamics of change in the probability variables can be obtained from the solution of the first-order equation:

$$
d y / d t=\alpha_{\mathrm{y}}^{*}(1-y)-\beta_{\mathrm{y}}^{*} y,
$$


where $y$ refers to $m^{*}, h^{*}$ and $n^{*}$, respectively. The activation, $\alpha_{\mathrm{y}}^{*}$, and deactivation, $\beta_{\mathrm{y}}^{*}$, parameters for smooth muscle satisfy the following relations:

$$
\begin{aligned}
& \alpha_{m}^{*}=0.005\left(\varphi_{(c, l)}-\bar{\varphi}_{m}\right) /\left(\exp \left(0.1\left(\varphi_{(c, l)}-\bar{\varphi}_{m}\right)\right)-1\right), \beta_{m}^{*}=0.2 \exp \left(\left(\varphi_{(c, l)}+\bar{\varphi}_{m}\right) / 38\right), \\
& \alpha_{h}^{*}=0.014 \exp \left(-\left(\bar{\varphi}_{h}+\varphi_{(c, l)}\right) / 20\right), \quad \beta_{h}^{*}=0.2 /\left(1+\exp \left(0.2\left(\bar{\varphi}_{h}-\varphi_{(c, l)}\right)\right)\right), \\
& \left.\alpha_{n}^{*}=0.006\left(\varphi_{(c, l)}-\bar{\varphi}_{n}\right) /\left(\exp \left(0.1\left(\varphi_{(c, l)}-\bar{\varphi}_{n}\right)\right)-1\right), \quad \beta_{n}^{*}=0.75 \exp \left(\bar{\varphi}_{n}-\varphi_{(c, l)}\right)\right),
\end{aligned}
$$

The dynamics of propagation of the electrical wave, $\varphi_{c}$, along the isotropic circular muscle layer is:

$$
C_{m} \frac{\partial \varphi_{c}}{\partial t}=\frac{M_{v s}}{1+\mu_{\tilde{s}_{1}}}\left\{\frac{\partial}{\partial \tilde{s}_{1}}\left(\frac{g_{0, \tilde{s}_{1}}^{*}}{\lambda_{c}} \frac{\partial \varphi_{c}}{\partial \tilde{s}_{1}}\right)+\frac{\partial}{\partial \tilde{s}_{2}}\left(\frac{g_{0, \tilde{s}_{1}}^{*}}{\lambda_{l}} \frac{\partial \varphi_{c}}{\partial \tilde{s}_{2}}\right)\right\}-I_{\text {ionic }}^{*}
$$

here the above-mentioned abbreviations and relationships for $I_{\text {ionic }}^{*}$ are used.

Plastic pellets which are used to study the gut transit time have the form of a sphere or ellipsoid. We assume that during all stages of propulsion the wall is in contact with the pellet. Its motion is due to the propagation of the electromechanical wave of deformation along the FU and is subjected to dry and viscous friction. The contact forces between the wall of the bioshell and the pellet are orthogonal to the surface of the pellet.

The kinematic equation of the motion of the bolus is governed by:

$$
\eta \frac{d Z_{c}}{d t}+F_{d}=\int_{z_{1}}^{z_{2}} \int_{0}^{r} F_{c} d z d \zeta
$$

where: $F_{c}, F_{d}$ are the contact force and the force of dry friction, respectively, $\eta$ is the coefficient of viscous friction. During all stages of dynamic reaction the points of the wall lie over the surface of the sphere:

$$
\mathfrak{K}_{c}=\left\{\left(Z_{c}-u_{z}\right)^{2}+\left(r_{0}+u_{r}\right)^{2}+\left(r_{0}+u_{s}\right)^{2}-R^{2}<0\right\}, \quad z \in\left[z_{1}, z_{2}\right]
$$

where: $\boldsymbol{u}=\left(u_{z}, u_{r}, u_{s}\right)$ - the displacement vector; $Z_{c}-$ the position of the center of the sphere at time $t ; z_{1}, z_{2}-$ the boundary points of contact of the sphere and the wall.

The initial conditions assume that the system is in the resting state. The boundary conditions are: i) the excitation of the system is due a discharge of the interstitial cell of Cajal (ICC-MP) of a known intensity, ii) the oral (left) end of the bioshell is rigidly fixed, iii) the aboral (right) end expands by the propagating 
bolus. The application of Lotronex and Zelnorm effects the mechanisms of synaptic neurotransmission via activation of the 5-HT3 and 4 type receptors located on the smooth muscle membrane.

The finite difference schemes of second order of accuracy with respect to spatial and time variables have been used to solve the system of equations.

\section{Results}

In the resting state, a segment of the gut maintains its initial cylindrical form under the effect of an intraluminal pressure and an enclosed rigid body. There are small variations in the concentration of intracellular calcium and undulation of active forces in the muscle. The contact forces exerted on the surface of the pellet and the propulsive forces generated by the smooth muscle layers are insufficient to propel the pellet along the FU. The FU is excited by the discharge of a pacemaker cell ICC-MP, situated at the left boundary. The wave of excitation reaches smooth muscle and initiates the propagating waves of depolarization amplitude $78-82 \mathrm{mV}$ along the syncytia. They travel along the surface of the muscle layers at a constant velocity $0.45-0.5 \mathrm{~cm} / \mathrm{s}$.

The elevation of the concentration of intracellular calcium initiates a cascade of intracellular contractile mechanisms leading to the development of active forces of contraction. The wave of contraction occurs in the longitudinal syncytium and has amplitude $14.7 \mathrm{mN} / \mathrm{cm}$, a wavelength, $0.45 \mathrm{~cm}$, and propagates aborally at $0.4 \mathrm{~cm} / \mathrm{s}$. The force generated by the circular syncytium has the maximum amplitude $17.8 \mathrm{mN} / \mathrm{cm}$, a wavelength $0.52 \mathrm{~cm}$, and propagates at a speed $0.35 \mathrm{~cm} / \mathrm{s}$. Because of the intrinsic nonlinearity of electromechanical coupling within the smooth muscle syncytia there is no symmetry in the dynamics of the stress-strain distribution along the inner and outer layers. In contrast, the collagenous and elastin fibers of the weight bearing stroma of the biocomposite redistribute the load uniformly along the surface of the bioshell (Fig. 1).

The maximum force in the longitudinal layer is $89 \mathrm{mN} / \mathrm{cm}$ and in the circular $-1851 \mathrm{mN} / \mathrm{cm}$. There is an omnipresent phase lag between the propagating waves of contraction-relaxation. Thus the electromechanical wave in the longitudinal layer precedes the wave in the circular one. This pattern of mechanical activity corresponds to the propulsion of the pellet along the segment of the gut (Fig. 2). The movement of the pellet in the aboral direction concurs with the dynamics of the propagation of the wave of depolarization along the circular syncytium. The average velocity of propagation is $0.8 \mathrm{~cm} / \mathrm{s}$. However, it fluctuates at different moments of the process. This explains a physiological phenomenon of "mixing" that takes place along with the propulsion.

\subsection{Effect of Lotronex (GlaxoSmithKline)}

We studied numerically the effects of the drug, Lotronex, to treat irritable bowel syndrome. The pharmacological mechanism of action of Lotronex is 5-HT type 3 receptor antagonism. The latter have ionotropic modulatory effect on $\mathrm{Ca}^{2+}$ - 
activated $K^{+}$and potential sensitive $K^{+}$channels in the system. The effects of the drug were achieved by altering values of $g_{C a(K)}$ and $g_{K}\left(\mathrm{mSm} / \mathrm{cm}^{2}\right)$. The administration of Lotronex hyperpolarizes the smooth muscle syncytium. The above changes cause a significant reduction in the force of contraction in the longitudinal $(70 \mathrm{mN} / \mathrm{cm})$ and circular $(1345 \mathrm{mN} / \mathrm{cm})$ smooth muscle layers. These lead to impairment of propulsive activity of the pellet of the gut. The recorded average velocity of the pellet is $0.23 \mathrm{~cm} / \mathrm{s}$.

\subsection{Effect of Zelnorm (Novartis, AB)}

The suggested mechanism of action of Zelnorm is 5HT type 4 receptor agonist. The application of Zelnorm restores the reciprocal relationship in contractionrelaxation of the longitudinal and circular smooth muscle layers, which is an essential component in normal propulsive activity of the gut. The results of numerical simulations show a slight decrease in transit time of the pellet. The velocity of propulsion is constant throughout and equals $0.4 \mathrm{~cm} / \mathrm{s}$. An increase in tone of the circular smooth muscle layer to $2050 \mathrm{mN} / \mathrm{cm}(1851 \mathrm{mN} / \mathrm{cm}$ is the normal recorded value) is observed, while there is an insignificant rise in the total force in the longitudinal layer. However, the pattern of propulsive activity of the segment demonstrates an even expulsion of the pellet without "mixing".

\section{Discussion}

The model presented here is an elaboration of the previous research on mathematical modeling of gastrointestinal tract [1-3]. A novel fundamental concept of a functional unit is successfully implemented in the construction of the model of pellet propulsion. It integrates, biomechanical properties and regulatory electrophysiological processes of an isolated segment of the gut and thus reproduces accurately combined events of: i) propagation of the wave of depolarization along the longitudinal and circular smooth muscle layers of the gut wall with electromechanical conjugation and the development of forces of contraction-relaxation, ii) shape changes, and iii) propulsion of the pellet (bolus).

The results of numerical simulations were validated against the data obtained in vivo and in vitro [4 -7]. Thus the results of simulations of the propulsion of the pellet in a segment show that the velocity of the propagation varies between $0.4-1.13 \mathrm{~cm} / \mathrm{s}$. According to D'Antona et al, an average velocity of the transit of a metal spherical bid in an isolated segment of the colon of a guinea pig is $0.75-0.8 \mathrm{~cm} / \mathrm{s}$. Movements of the pellet are caused by strong contractions of the circular smooth muscle layer. Based on a qualitative analysis of the experimental data, which show the development of a ring contraction with the narrowing of the lumen gut proximal to the metal bit, we can assume that it corresponds to the peak of the total force $(1851 \mathrm{mN} / \mathrm{cm})$ distribution observed in our results [4]. Our results fall in the range of experimentally measured velocity of the movement of intraluminal content $(1-3 \mathrm{~cm} / \mathrm{s})$, which was observed in the studies of colonic motility in humans [8-9]. 

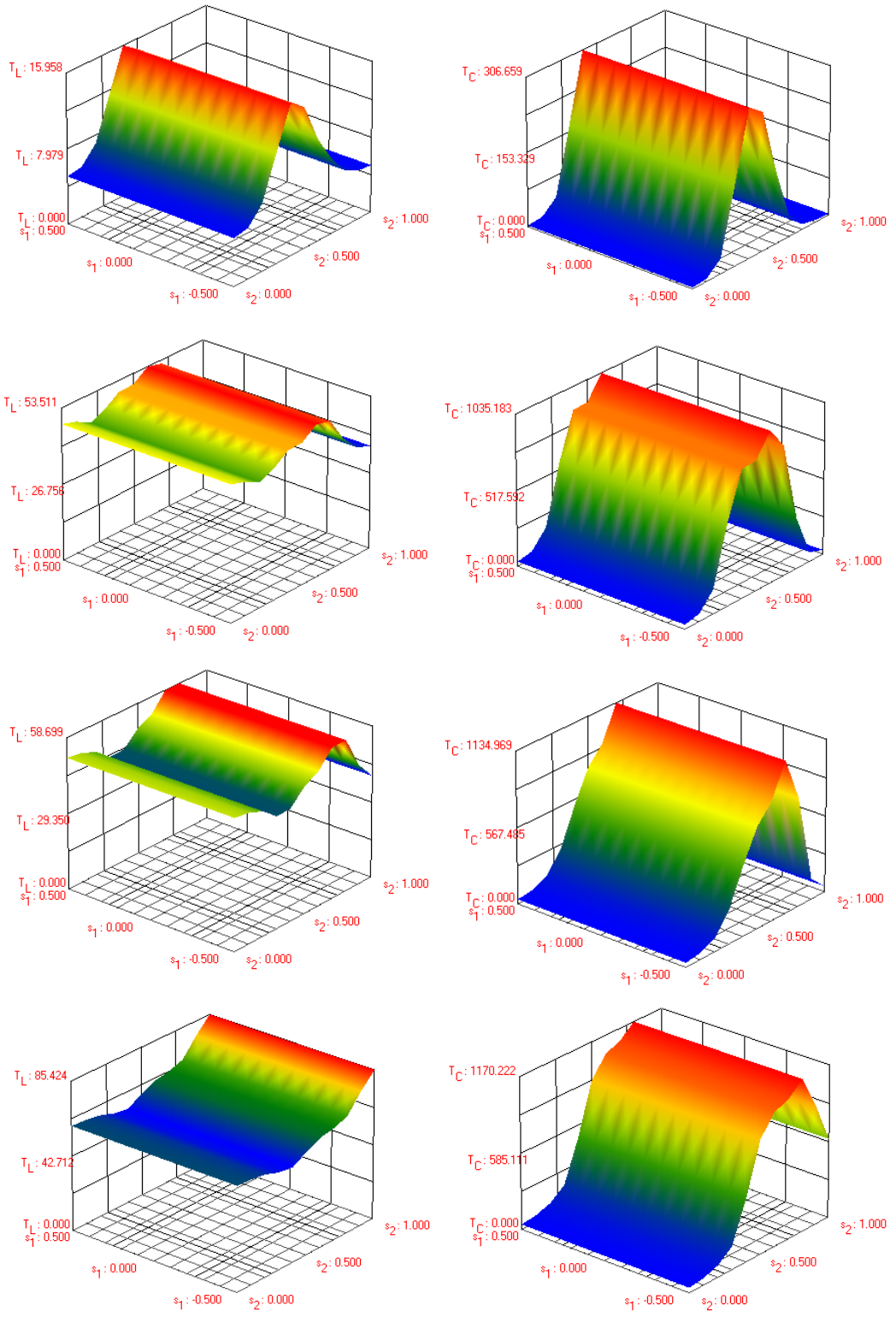

Figure 1: Development of total forces in the longitudinal (right column) and circular (left) smooth muscle syncytia in the FU. 

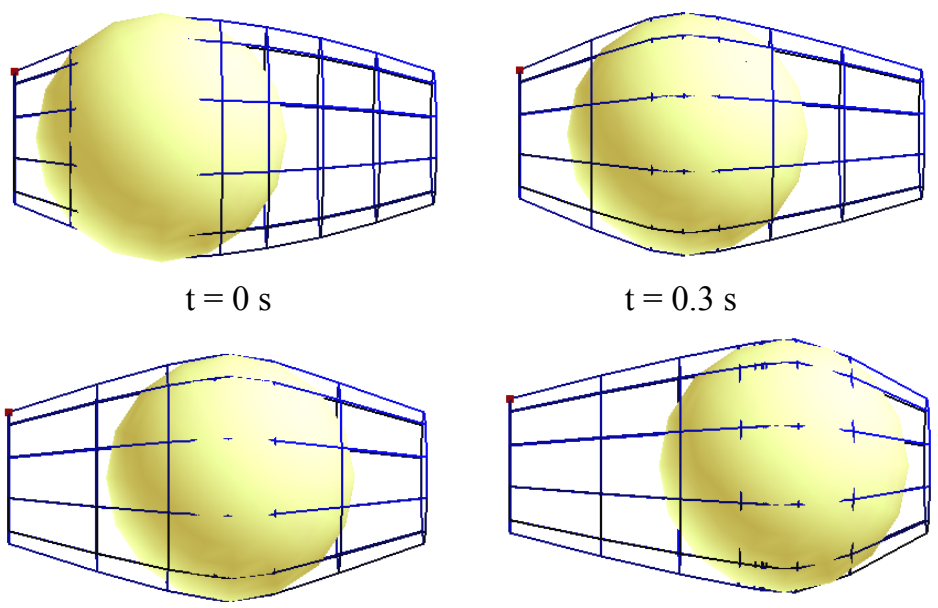

$\mathrm{t}=0.6 \mathrm{~s}$

$\mathrm{t}=0.9 \mathrm{~s}$

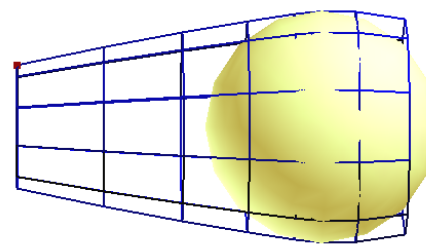

$\mathrm{t}=1.2 \mathrm{~s}$

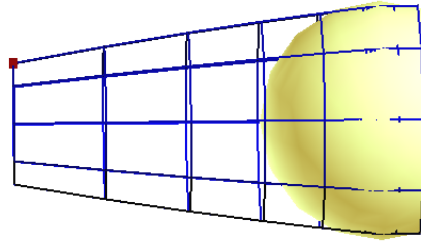

$\mathrm{t}=1.5 \mathrm{~s}$

Figure 2: Dynamics of the propagation of the pellet at times as indicated.

The model allows us to reproduce the effects of drugs affecting gut motility. We numerically simulated the mechanism of action of the once popular bowel drug, Lotronex, to treat irritable bowel syndrome and analyzed its most prominent side effect - severe constipation - that led to hospitalization, surgical treatment and the fatalities of a number of patients. Administration of the drug causes a $33 \%$ reduction $(1345 \mathrm{mN} / \mathrm{cm})$ in the total force of contraction and a dilatation of a segment of the gut. This significantly slows down the propulsion of the pellet which moves at a velocity $0.23 \mathrm{~cm} / \mathrm{s}$. This result correlates with the clinical studies [6-7] that demonstrate decrease in colonic transit.

Treatment of the functional unit with Zelnorm slightly decreases the transit time of the pellet, increases the total force of contraction of the circular smooth muscle layer by $11 \%$, and changes the pattern of propulsion, which becomes even without "mixing". That may explain low clinical effectiveness of the drug, (only 10-12\% over the effect of the placebo), to treat constipation [6].

Although the model answers many questions related to normal and pathological physiology of gut motility further improvements of its basic concepts and mathematical formulation are needed. For example, to enable study 
of the mechanisms of neuroneuronal and neuromusclular signal transduction, the phenomena of receptor polymodality and neurotransmitter co-localization and co-transmission should be included in the model. Another interesting opportunity is in elaboration of sensory pathways of the gut and the addition of chemoreceptors and nociceptors into the system. Also high level regulatory mechanisms, the prevertebral ganglia and the central nervous system, are not integrated in the model. The above proposed modifications would increase the biological accuracy of the model and would allow a quantitative study of the intrinsic neurobiological processes of motility, which are not accessible by any currently existing experimental techniques.

\section{References}

[1] Miftakhov, R. \& Abdusheva, G.R. Small bowel propulsion: transport of a solid bolus. Biomedical and Life Physics, D. Ghista (Ed), Berlin, Springer, pp. 253-260, 1993

[2] Miftakhov, R. \& Vannier, M. Nonlinear dynamic waves in electromechanical excitable biological media. Advances in Fluid Mechanics, M. Rahman, R. Verhoeven, C.A. Brebbia (Eds), WIT Press, Southampton, pp. 725-735, 2002

[3] Miftakhov, R. \& Fedotov, E.M. The concept of a functional unit of the gut. Advances in Fluid Mechanics V, A. Mendes, M. Rahman \& Carlos Brebbia (Eds), WIT Press, Southampton, pp. 553-561, 2004

[4] D'Antona, G., Hennig, G.W., Costa, M., Humphreys, C.M. \& Brookes, S.J.H. Analysis of motor patterns in the isolated guinea-pig large intestine by spatio temporal maps. Neurogastroenterology \& Motility, 13, pp. 483492, 2001

[5] Lacy, B.E. \& Yu S. Tegaserod. A new 5-HT4 agonist. Journal of Clinical Gastroenterology, 34(1), pp. 27-33, 2002

[6] Gunput, M.D. Review Article: clinical pharmacology of alosetron. Alimentary Pharmacology and Therapeutics, 13 (2), pp. 70-76, 2001

[7] Humphrey, P.P.A., Bountra, C., Clayton, N., \& Kozlowski, K. Review article: the therapeutic potential of 5-HT3 receptor antagonists in the treatment of irritable bowel syndrome. Alimentary Pharmacology and Therapeutics, 13 (2), pp. 31-38, 2001

[8] Krevsky, B.L., Malmud, L.S., D'Ercole, F., Maurer, A.H. \& Fisher, R.S. Colonic transit schintigraphy: a physiologic approach to the quantitative measurement of colonic transit in humans. Gastroenterology, 91, pp. 11021112,1986

[9] Marciani, L., Young, P., Wright, J., Moore, R., Coleman, N., Gowland, P.A. and Spiller, R.C. Antral motility measurements by magnetic resonance imaging. Neurogastroenterology \& Motility, 13, pp. 511-518, 2001 